\title{
Associations among Obesity Degree, Glycemic Status, and Risk of Heart Failure in 9,720,220 Korean Adults (Diabetes Metab J 2020;44:592-601)
}

\section{Darae Kim \\ Division of Cardiology, Department of Medicine, Heart Vascular Stroke Institute, Samsung Medical Center, Sungkyunkwan University School of Medicine, Seoul, Korea}

Heart failure (HF) is a growing public health concern with increasing prevalence worldwide. The estimated prevalence of HF is estimated to be $1.53 \%$ in 2013 , and is expected to double in 20 years in Korea [1]. Elevated body mass index (BMI) and insulin resistance are major risk factors for HF [2]. Recognition of individuals at risk for HF is critical because early treatment at the preclinical stage may prevent HF progression and improve outcomes.

Rhee et al. [3] analyzed the risk of HF according to baseline glycemic status and BMI in a Korean population using National Health Insurance Data. This nationwide population study showed a 1.86-fold increased risk of HF in diabetic patients compared to those with normoglycemia, which is consistent with previous data [4]. The incidence of HF increased with duration of diabetes. Risk of HF showed a J-shaped curve according to BMI regardless of baseline glycemic status. Those with class II obesity and those who were underweight had significantly increased risk for developing HF compared to normal and overweight groups. Rhee et al. [3] showed an overall trend among obesity degree, glycemic status, and HF in the Korean population. However, there are many unrevealed confounding factors that must be taken into account, such as sex, age, and subtypes of HF (preserved vs. reduced ejection fraction [EF], ischemic vs. non-ischemic, and chronic stable vs. acute decompensated). The results of this study raise important questions to be answered to appreciate clinical implications between BMI and HF, especially from therapeutic and prognostic perspectives.
HF is a clinical syndrome that results from structural or functional impairment of ventricular filling and/or ejection of blood [5]. HF is often classified according to EF; HF with reduced EF (HFrEF) vs. HF with preserved EF (HFpEF). The presenting symptoms of HFrEF and HFpEF are similar; however, the pathologic mechanisms are different, reflected by the finding that mortality reducing treatment in $\mathrm{HFrEF}$ failed to improve outcomes in HFpEF. A previous large populationbased study from the United States showed that obesity and associated cardiometabolic traits increase risk of HFpEF compared to that of HFrEF [6]. Notably, there was a different effect of obesity on HF subtypes between men and women. Among men, higher BMI was independently associated with both HF subtypes (hazard ratio [HR], 1.34; 95\% confidence interval [CI], 1.18 to 1.52 ; $P<0.0001$ for HFpEF) (HR, 1.24 ; $95 \% \mathrm{CI}$, 1.14 to $1.35 ; P<0.0001$ for HFrEF). On the contrary, among women, BMI was associated with incident HFpEF but not HFrEF (HR, 1.38 per 1-standard deviation; 95\% CI, 1.24 to 1.54, $P<0.0001$ for HFpEF) (HR, 1.09; 95\% CI, 0.96 to 1.24; $P=0.18$ for HFrEF; $P$ for difference=0.01). The J-shaped incidence curve of HF described by Rhee et al. [3] could change when HF subtype or sex is taken into account.

The risk of HF associated with obesity seems to be modifiable, suggesting another important question. Is there a therapeutic role of weight loss in obese patients in prevention of HF? Recent studies from Sweden have demonstrated that weight loss over longitudinal follow-up is associated with a lower risk of HF by comparing risk of HF in obese patients with and
Corresponding author: Darae Kim (D) https://orcid.org/0000-0003-3284-0904 Division of Cardiology, Department of Medicine, Heart Vascular Stroke Institute, Samsung Medical Center, Sungkyunkwan University School of Medicine, 81 Irwon-ro, Gangnam-gu, Seoul 06351, Korea

E-mail: daraekyrie@gmail.com
This is an Open Access article distributed under the terms of the Creative Commons Attribution Non-Commercial License (https://creativecommons.org/licenses/by-nc/4.0/) which permits unrestricted non-commercial use, distribution, and reproduction in any medium, provided the original work is properly cited. 
without bariatric surgery [7]. Also, a recent meta-analysis by Reddy et al. [8] demonstrated a hemodynamic effect of weight loss intervention in obese patients without HF. Weight loss in obese patients was associated with significantly reduced heart rate $(-9$ beats/min), mean arterial pressure $(-7 \mathrm{~mm} \mathrm{Hg})$, resting oxygen consumption, and reduced left and right heart filling pressures. This finding suggests that weight loss could attenuate hemodynamic derangement that can lead to HF, especially HFpEF. However, future prospective trials designed to evaluate the efficacy of weight loss intervention on modifying risk of HF are needed. Based on previous studies and growing prevalence of obesity and HF, more evidence is warranted to demonstrate the efficacy of weight loss intervention to prevent HF in different subgroups of the population. The previous study by Rhee et al. [3] provides a good starting point.

\section{CONFLICTS OF INTEREST}

No potential conflict of interest relevant to this article was reported.

\section{REFERENCES}

1. Lee JH, Lim NK, Cho MC, Park HY. Epidemiology of heart failure in Korea: present and future. Korean Circ J 2016;46:65864.

2. Kenchaiah S, Evans JC, Levy D, Wilson PW, Benjamin EJ, Larson MG, Kannel WB, Vasan RS. Obesity and the risk of heart failure. N Engl J Med 2002;347:305-13.

3. Rhee EJ, Kwon H, Park SE, Han KD, Park YG, Kim YH, Lee WY. Associations among obesity degree, glycemic status, and risk of heart failure in 9,720,220 Korean adults. Diabetes Metab J 2020;44:592-601.

4. Nichols GA, Gullion CM, Koro CE, Ephross SA, Brown JB. The incidence of congestive heart failure in type 2 diabetes: an update. Diabetes Care 2004;27:1879-84.

5. Yancy CW, Jessup M, Bozkurt B, Butler J, Casey DE Jr, Colvin MM, Drazner MH, Filippatos GS, Fonarow GC, Givertz MM, Hollenberg SM, Lindenfeld J, Masoudi FA, McBride PE, Peterson PN, Stevenson LW, Westlake C. 2017 ACC/AHA/HFSA focused update of the 2013 ACCF/AHA guideline for the management of heart failure: a report of the American College of Cardiology/American Heart Association task force on clinical practice guidelines and the Heart Failure Society of America. J Card Fail 2017;23:628-51.

6. Savji N, Meijers WC, Bartz TM, Bhambhani V, Cushman M, Nayor M, Kizer JR, Sarma A, Blaha MJ, Gansevoort RT, Gardin JM, Hillege HL, Ji F, Kop WJ, Lau ES, Lee DS, Sadreyev R, van Gilst WH, Wang TJ, Zanni MV, Vasan RS, Allen NB, Psaty BM, van der Harst P, Levy D, Larson M, Shah SJ, de Boer RA, Gottdiener JS, Ho JE. The association of obesity and cardiometabolic traits with incident HFpEF and HFrEF. JACC Heart Fail 2018;6:701-9.

7. Persson CE, Bjorck L, Lagergren J, Lappas G, Giang KW, Rosengren A. Risk of heart failure in obese patients with and without bariatric surgery in Sweden: a registry-based study. J Card Fail 2017;23:530-7.

8. Reddy YNV, Anantha-Narayanan M, Obokata M, Koepp KE, Erwin P, Carter RE, Borlaug BA. Hemodynamic effects of weight loss in obesity: a systematic review and meta-analysis. JACC Heart Fail 2019;7:678-87. 